\title{
Influence of tissue fixation on depth-resolved birefringence of oral cavity tissue samples
}

\author{
Karol Karnowski, ${ }^{\mathrm{a}, \mathrm{b}, *, \dagger}$ Qingyun Li, ${ }^{\text {a, }}$ Anima Poudyal, ${ }^{\mathrm{c}}$ Martin Villiger, ${ }^{\mathrm{d}}$ \\ Camile S. Farah, ${ }^{\text {ce,f }}$ and David D. Sampson ${ }^{\text {g,h }}$ \\ ${ }^{a}$ The University of Western Australia, Optical+Biomedical Engineering Laboratory, \\ Department of Electrical, Electronic, and Computer Engineering, Perth, \\ Western Australia, Australia \\ ${ }^{\text {b} P o l i s h ~ A c a d e m y ~ o f ~ S c i e n c e s, ~ I n s t i t u t e ~ o f ~ P h y s i c a l ~ C h e m i s t r y, ~ W a r s a w, ~ P o l a n d ~}$ \\ ${ }^{\mathrm{c}}$ The University of Western Australia, UWA Dental School, Perth, Western Australia, Australia \\ ${ }^{\mathrm{d} H a r v a r d}$ Medical School, Massachusetts General Hospital, Wellman Center for Photomedicine, \\ Boston, Massachusetts, United Sates \\ ${ }^{\mathrm{e} A u s t r a l i a n}$ Centre for Oral Oncology Research and Education, Perth, Western Australia, \\ Australia \\ ${ }_{\mathrm{f}}^{\mathrm{Fiona}}$ Stanley Hospital, Oral, Maxillofacial, and Dental Surgery, Murdoch, \\ Western Australia, Australia \\ ${ }^{\mathrm{g}}$ University of Surrey, Surrey Biophotonics, School of Physics, Guilford, United Kingdom \\ ${ }^{h}$ University of Surrey, Surrey Biophotonics, School of Biosciences and Medicine, Guilford, \\ United Kingdom
}

\begin{abstract}
Significance: To advance our understanding of the contrast observed when imaging with polarization-sensitive optical coherence tomography (PS-OCT) and its correlation with oral cancerous pathologies, a detailed comparison with histology provided via ex vivo fixed tissue is required. The effects of tissue fixation, however, on such polarization-based contrast have not yet been investigated.
\end{abstract}

Aim: A study was performed to assess the impact of tissue fixation on depth-resolved (i.e., local) birefringence measured with PS-OCT.

Approach: A PS-OCT system based on depth-encoded polarization multiplexing and polarization-diverse detection was used to measure the Jones matrix of a sample. A wide variety of ex vivo samples were measured freshly after excision and $24 \mathrm{~h}$ after fixation, consistent with standard pathology. Some samples were also measured $48 \mathrm{~h}$ after fixation.

Results: The tissue fixation does not diminish the birefringence contrast. Statistically significant changes were observed in 11 out of 12 samples; these changes represented an increase in contrast, overall, by $11 \%$ on average.

Conclusions: We conclude that the fixed samples are suitable for studies seeking a deeper understanding of birefringence contrast in oral tissue pathology. The enhancement of contrast removes the need to image immediately postexcision and will facilitate future investigations with PS-OCT and other advanced polarization-sensitive microscopy methods, such as mapping of the local optic axis with PS-OCT and PS-optical coherence microscopy.

(C) The Authors. Published by SPIE under a Creative Commons Attribution 4.0 Unported License. Distribution or reproduction of this work in whole or in part requires full attribution of the original publication, including its DOI. [DOI: 10.1117/1.JBO.25.9.096003]

Keywords: biomedical imaging; tissue fixation; optical coherence tomography; oral tissue.

Paper 200071R received Mar. 17, 2020; accepted for publication Jul. 29, 2020; published online Sep. 10, 2020.

\footnotetext{
*Address all correspondence to Karol Karnowski, E-mail: karol.karnowski@uwa.edu.au

${ }^{\dagger}$ These authors contributed equally to this work.
} 


\section{Introduction}

Even though the potential of using optical coherence tomography (OCT) in the oral cavity and the oropharynx has been reported in a positive light, ${ }^{1-3}$ the evidence suggests that differentiation between healthy and potentially malignant oral tissue is still challenging when only conventional OCT image contrast is available. Some studies have been limited to only a few samples ${ }^{2}$ or have identified disease from a single feature extracted from OCT scans (e.g., epithelial thickness). ${ }^{1}$ In most studies reported thus far, diagnostic scores have been based on image comparison (OCT versus histology) by a previously trained surgeon and/or pathologist. ${ }^{3-9}$ The sensitivity and specificity of differentiating pathological from normal tissue reported in those studies are in the ranges $81.5 \%$ to $93.1 \%$ and $78 \%$ to $97.3 \%$, respectively. The detection of carcinoma in situ, squamous cell carcinoma (SCC) versus noncancer (sensitivity: 93.1\% and specificity: 93.1\%), or SCC versus other pathologies (sensitivity: $93.1 \%$ and specificity: $97.3 \%$ ) have also been reported. ${ }^{7}$ The study by Kraft et al. ${ }^{10}$ suggests the exact grading of oral dysplasia might be better determined if microlaryngoscopy is supported by examination by OCT. It is not clear, however, what the diagnostic accuracy would be if only OCT images were analyzed. The only study assessing the automatic classification of oral malignancy from OCT images alone was performed in an animal model (hamster cheek pouch). ${ }^{11}$ The performance of the sophisticated method used was failure-prone because of common artifacts in OCT images (e.g., detector saturation due to specular reflection).

Various extensions have been proposed to enhance the contrast in OCT images in general. For instance, compression OCT elastography (OCE) shows promise as a tool for interoperative assessment of cancer margins for ex vivo human breast samples. ${ }^{12}$ However, in vivo OCE imaging in the oral cavity with controllable tissue compression remains an intriguing prospect, which has not yet been reported. Polarization-sensitive OCT (PS-OCT) has demonstrated polarization contrast between uninvolved stroma and tumor ${ }^{13,14}$ and, by contrast, does not require tissue compression. Various studies using PS-OCT on healthy and pathologically altered animal $^{15}$ and human ${ }^{16-20}$ oral tissue samples have revealed promising enhanced contrast provided by differences in the polarization properties of oral cavity tissue structures. An important recent advance is the refinement of reconstruction methods to enable extraction of local tissue birefringence (localized in depth), which has led to marked improvements in contrast and fidelity with tissue morphology, including in (healthy) oral cavity tissues. ${ }^{21}$

To further advance our understanding of contrast assessed by PS-OCT and its potential in oral cancer diagnosis and treatment, a detailed comparison with corresponding histology is needed. PS-OCT measurement directly after excision is not always feasible and investigation of fixed samples at a later time, if feasible, may be preferred. Work by Grieve et al. ${ }^{22}$ reported that tissue fixation may diminish the visibility of some features when measured with OCT. That study, however, investigated the fixation of retina samples and was performed with OCT intensity without insight into the polarization effects of the fixation process. No studies on the effects of tissue fixation on PS-OCT imaging have been presented to date. The closest research that we have found on the effects of fixation on tissue optical polarization properties was performed with optical polarimetry. ${ }^{23}$ Thin sections of porcine liver and heart were measured in transmission mode and an overall increase of birefringence upon fixation was reported. However, the authors cautioned that polarization contrast for other tissue types could not be extrapolated from their study and should be determined independently.

In this study, the effect of tissue fixation on local birefringence contrast determined with PS-OCT was assessed. Ex vivo oral cavity samples were measured immediately postexcision, then fixed in formalin solution and measured again $24 \mathrm{~h}$ after the commencement of fixation, following standard pathology. Some samples were measured $48 \mathrm{~h}$ after fixation to confirm the results. To the best of our knowledge, this is the first time such an investigation of PS-OCT imaging contrast has been performed. The results suggest that tissue fixation does not substantially alter local birefringence contrast and open up opportunities for broader studies based on a variety of pathological precancerous and cancerous oral tissue samples. Such studies should improve prospects for the in vivo differentiation of normal and abnormal tissue based on PS-OCT. 


\section{Materials and Methods}

\subsection{Specimen Preparation}

The imaging was performed at the Oral Medicine Clinic, Sir Charles Gairdner Hospital (Perth, Western Australia). The study was approved by the Human Research Ethics Office of the University of Western Australia (UWA Ethics number RA/4/1/8562) and written informed consent was obtained from all participants. Over a 3-day period, we measured 12 freshly excised tissue samples from different locations in the oral cavity and covering a range of pathologies (see Table 1 for the clinical presentation of excised samples). Excised samples were transferred from the surgery to a nearby office and imaged with PS-OCT within $5 \mathrm{~min}$ of excision. Following the PS-OCT measurement, samples were immersed in a fixative solution (10\% neutral-buffered formalin) and stored at room temperature. The measurement of all stored samples was repeated at $24 \mathrm{~h}$ from excision $( \pm 45 \mathrm{~min})$. In routine pathology processing of ex vivo oral cavity tissue, samples of the size used in this study undergo 24-h fixation prior to further processing; therefore, we assumed $24 \mathrm{~h}$ was sufficient elapsed time for samples to be fully fixed. For reference, Hsiung et al. ${ }^{24}$ reported $18 \mathrm{~h}$ as the time necessary to ensure complete tissue fixation for hamster cheek pouch samples. Additionally, for further confirmation of fixation status, five samples were also measured at $48 \mathrm{~h}$ after excision.

\subsection{Polarization-sensitive OCT}

Imaging was performed with a custom PS-OCT scanner developed in-house and used previously for needle-based measurements of breast samples ${ }^{13}$ and adapted recently for bulk optics-based measurements. ${ }^{24}$ The technical specifications of the scanner are summarized as follows. The system operated with an A-line repetition rate of $50 \mathrm{kHz}$ at the center wavelength of $1310 \mathrm{~nm}$ and a scanning range of $110 \mathrm{~nm}$. We employed passively depth-encoded polarization multiplexing and polarization-diverse detection to measure the full Jones matrix containing the cumulative polarization information of backscattered light after round-trip propagation through the sample. ${ }^{26}$ To provide sufficient imaging range for depth-encoded multiplexing, a custommade sampling clock frequency-doubling circuit was used. The resolution of the system was $13 \mu \mathrm{m}$ and $20 \mu \mathrm{m}$ in tissue in the axial (assuming a tissue refractive index $n=1.4^{27}$ ) and lateral directions, respectively. Single volumetric data sets consisting of $1000 \times 1000$ A-scans covering

Table 1 Ex vivo samples used for the study described by site in the oral cavity and clinical presentation.

\begin{tabular}{lll}
\hline \hline$\#$ & \multicolumn{1}{c}{ Site } & \multicolumn{1}{c}{ Clinical presentation } \\
\hline 1 & Right ventral tongue & Squamous papilloma \\
2 & Right buccal mucosa & Leukoplakia \\
3 & Right soft palate & Leukoplakia \\
4 & Right buccal mucosa & Oral lichen planus \\
5 & RHS lateral tongue & Hyperkeratotic area adjacent to ulcer \\
6 & Left buccal mucosa & Oral lichen planus \\
7 & Lower left lip & Actinic cheilitis \\
8 & Left buccal mucosa & Oral lichen planus \\
9 & Left lateral tongue & Leukoplakia \\
10 & Left ventral tongue & Leukoplakia \\
11 & Right soft palate & Leukoplakia with pigmentation \\
12 & Right lower lip & Actinic cheilitis \\
\hline \hline
\end{tabular}


an area of $5.1 \times 5.1 \mathrm{~mm}$ with a depth range of $3.5 \mathrm{~mm}$ in air were captured within $20 \mathrm{~s}$ for each sample. Further technical details on the instrument (not relevant to the current study) can be found in Refs. 13 and 24. The $X Y$ scan area was sufficient to image the whole sample in the lateral dimensions.

Data processing to extract local birefringence is summarized as follows. Mueller-Jones matrices were constructed from the measured Jones matrices and spatially averaged before extraction of the local birefringence using a differential Mueller matrix algorithm. ${ }^{13}$ In contrast to the majority of previous studies, where average birefringence is computed from the cumulative retardance from the tissue surface to a given depth, here, we provide local birefringence at close to the same resolution as the OCT intensity images. Separately, in our recent in vivo study, ${ }^{17}$ we have shown that results presented by means of cumulative retardation provide lower contrast, especially at greater depths, compared with local birefringence. An averaging kernel of $26 \mu \mathrm{m}$ axially and $40 \mu \mathrm{m}$ laterally was used to improve the local birefringence contrast at the expense of a twofold reduction in spatial resolution. Both OCT intensity and local birefringence cross-sectional images are presented in grayscale in Figs. 1(a) and 1(b). Better insight regarding the features of measured samples is provided using an en face view [Figs. 1(c) and 1(d)]. The measured image data were first flattened by automatic subtraction of the extracted position of the tissue surface, so each en face slice corresponds to a given depth below the tissue surface (as marked by the red dotted curve on the cross-sections in Figs. 1(a) and 1(b). We note that, for some of the samples, the surface of the excised tissue does not correspond to the natural anatomical surface of the tissue before extraction. The flattening procedure was introduced to create en face slices and to enable automation of the quantitative analysis.

Surface flattening was performed and en face slices were prepared for all measurements. The orientation angle (in the $X Y$ plane) of the same sample was found to differ between measurements for some samples. This was irrelevant for quantitative analysis of image contrast. However, for visual presentation and comparison purposes, the orientation angle difference was manually removed in postprocessing. The angle registration was performed by visual inspection based on sample topography features (see Figs. 1-3).

We automated the quantitative analysis of local birefringence contrast, thereby avoiding observer bias. We selected data for analysis using a binary mask (separate masks were created
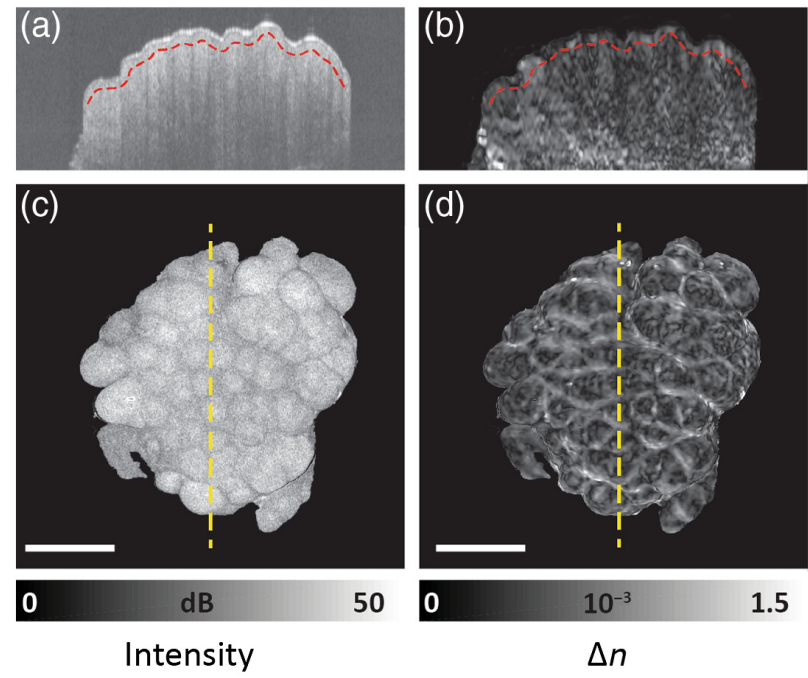

Fig. 1 Cross-sectional (B-scan) PS-OCT images of the ex vivo right ventral tongue sample (sample \#1 from Table 1): (a) conventional OCT intensity image, (b) depth-resolved birefringence $(\Delta n)$ revealing increased contrast compared to OCT intensity image, (c) en face slices from the depth indicated by the red dotted curve in (a), and (d) depth-resolved birefringence from the depth indicated by the red dotted curve in (b). Yellow dotted lines correspond to the position of crosssectional images. For images on (b)-(c), regions corresponding to low SNR, as measured in the conventional OCT images, were masked out. Images were scaled in depth assuming a refractive index of $n=1.4$. Scale bar: $1 \mathrm{~mm}$. 

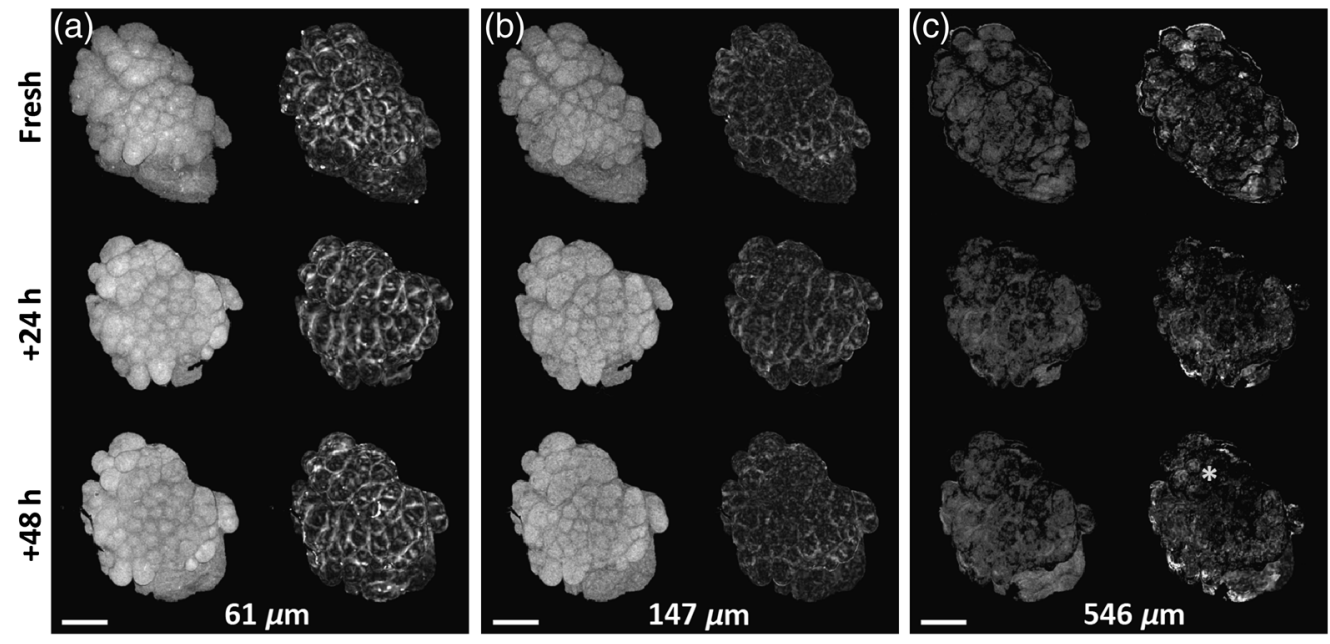

Fig. 2 Paired-OCT intensity on the left and birefringence on the right-en face slices of an ex vivo right ventral tongue sample (sample \#1 from Table 1) at various depths (a)-(c) for freshly excised tissue, and $24 \mathrm{~h}$, and $48 \mathrm{~h}$ after fixation was initiated. Scale bar: $1 \mathrm{~mm}$. The yellow asterisk indicates an area where birefringence contrast is locally reduced. The OCT intensity and birefringence scale bars are the same as in Fig. 1 and omitted here for clarity.

for each en face depth slice and each time point) by thresholding of OCT intensity data followed by two morphological operations: (1) closing of the image to fill the gaps in the mask and (2) erosion of the image to remove bright pixels not corresponding to the mask, applied to the mask to eliminate noise and irrelevant artifacts. The intensity-based masks were used on the corresponding en face slices of local birefringence. All procedures were optimized on one randomly selected data set, visually tested on three additional data sets, and then applied in unsupervised processing of all data sets. The contrast was calculated for each en face slice, starting from the surface to a depth of almost $800 \mu \mathrm{m}$, determined as the ratio of the standard deviation $\left(\sigma_{\Delta n}\right)$ to mean value of birefringence $(\overline{\Delta n})$ (after application of the averaging kernel):

$$
C_{\Delta n}=\frac{\sigma_{\Delta n}}{\overline{\Delta n}}
$$

For samples \#9 and \#11, the analysis depth was reduced to 600 and $450 \mu \mathrm{m}$, respectively, as no sample-related signal was present below these depths.

For each sample and time point, we performed a statistical comparison for every en face slice $(N=112)$ between measurements of freshly excised samples and fixed samples measured $24 \mathrm{~h}$ (and $48 \mathrm{~h}$ where available) after the commencement of fixation. The statistical comparisons were first tested for normality of the distribution using the Shapiro-Wilk test. ${ }^{28}$ As all pairs were nonnormally distributed, the Wilcoxon test ${ }^{29}$ with $5 \%$ significance level was used. Additionally, we analyzed the contrast differences at given depths in the complete sample population. For every depth slice, we calculated the contrast differences between the fresh and 24-h time points as well as between the fresh and 48-h time points. The mean difference and standard deviation for each slice were calculated and plotted versus depth.

\section{Results}

\subsection{Local Birefringence Qualitative Contrast}

Figure 2 shows en face OCT intensity and local birefringence sections extracted from different depths below the sample surface at the different time points of the fixation process. The sample was excised from the right ventral tongue and was preassessed as squamous papilloma. For this sample, we observe high variability of birefringent structures, which most likely represent highly aligned collagen fibers in the reticular layer, as described in our previous study. ${ }^{21}$ In general, in 

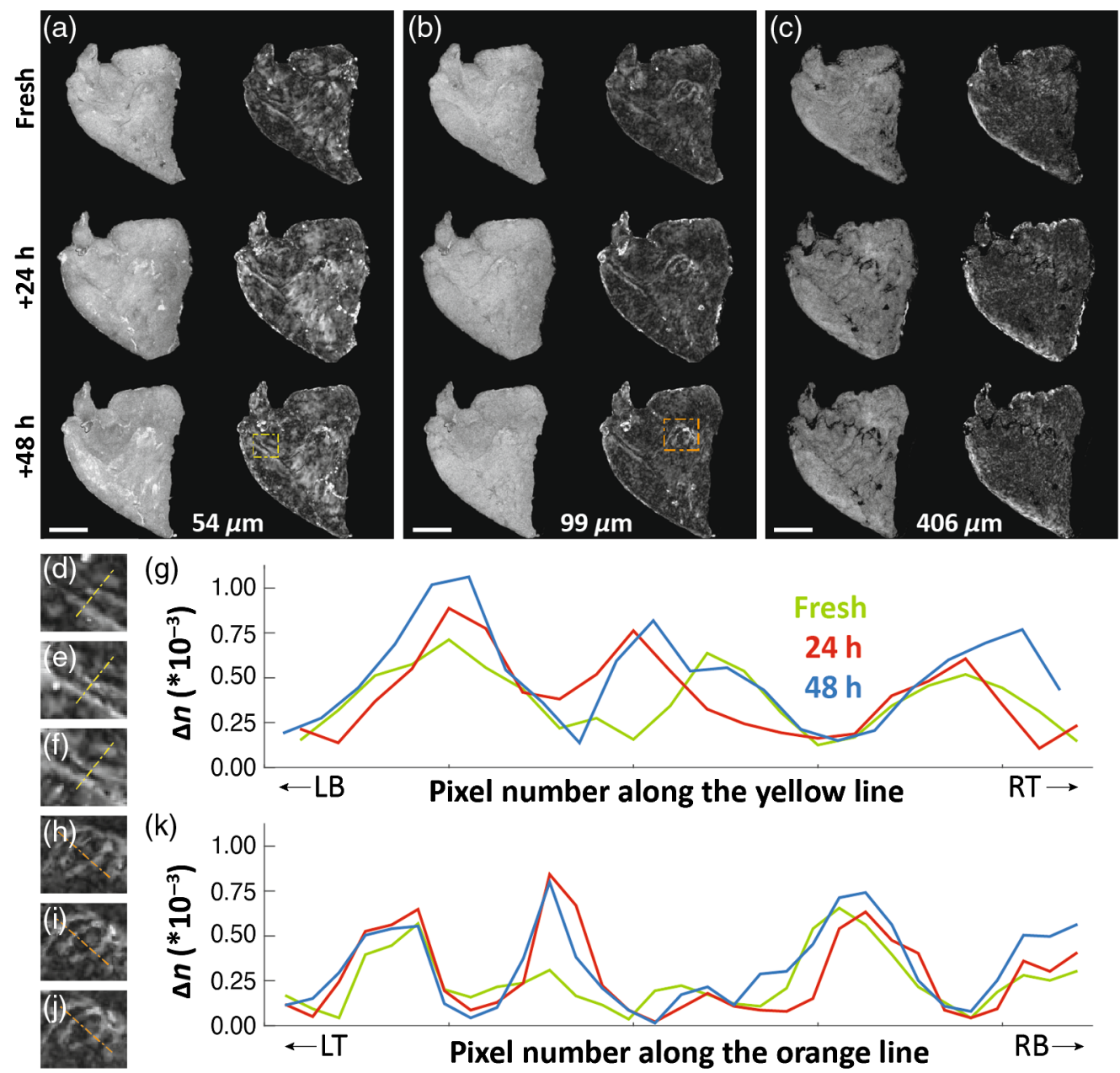

Fig. 3 Paired-OCT intensity on the left and birefringence on the right-en face slices of the ex vivo right soft palate sample (sample \#3 from Table 1) at various depths (a)-(c) for freshly excised tissue as well as $24 \mathrm{~h}$ and $48 \mathrm{~h}$ after fixation was initiated. The yellow and orange dashed-line boxes indicate the regions further visualized and analyzed in (d)-(k). Scale bar: $1 \mathrm{~mm}$. Abbreviations used in $(\mathrm{g})$ and $(\mathrm{k})$ : LB, left bottom and RT, right top related to the yellow dotted line on zoomed birefringence maps (d)-(f), LT, left top and RB, right bottom for similar orange dotted line $(\mathrm{h})-(\mathrm{j})$. The OCT intensity and birefringence scale bars are the same as in Fig. 1 and omitted here for clarity.

Fig. 2, we observe the contrast in both intensity and birefringence due to tissue alteration induced by the fixation procedure. For the deepest image sections [Fig. 2(c)], some examples of decreased contrast are observed (e.g., at the location marked by the yellow asterisk). This may be due to the effect of axial tissue shrinkage during fixation causing previously deeper layers to be shifted closer to the surface. The overall and rigorous assessment of the birefringence contrast change, however, is difficult based on visual inspection alone; therefore, we undertook the quantitative analysis described above and presented in the following sections.

The second example [Figs. 3(a)-3(c)] taken from the right soft palate reveals changes in the pattern of birefringent features. Magnified views [Figs. 3(d)-3(j)] depict visible improvement in the birefringence contrast during the fixation process. Two regions (yellow and orange boxes in Figs. 3(a) and 3(b) were selected to show the effect quantitatively. Zoomed maps of birefringence for freshly excised [Figs. 3(d) and 3(h)], 24 h [Figs. 3(e) and 3(i)], and 48 h [Fig. 3(f) and 3(j)] after fixation, as well as plots of birefringence extracted along selected lines [Figs. 3(g) and 3(k)], are presented. We chose to present data from the soft palate sample (Fig. 3) at different depths compared to the ventral tongue sample (Fig. 2) to help better appreciation of the variations in sample birefringence due to fixation, including with depth. 


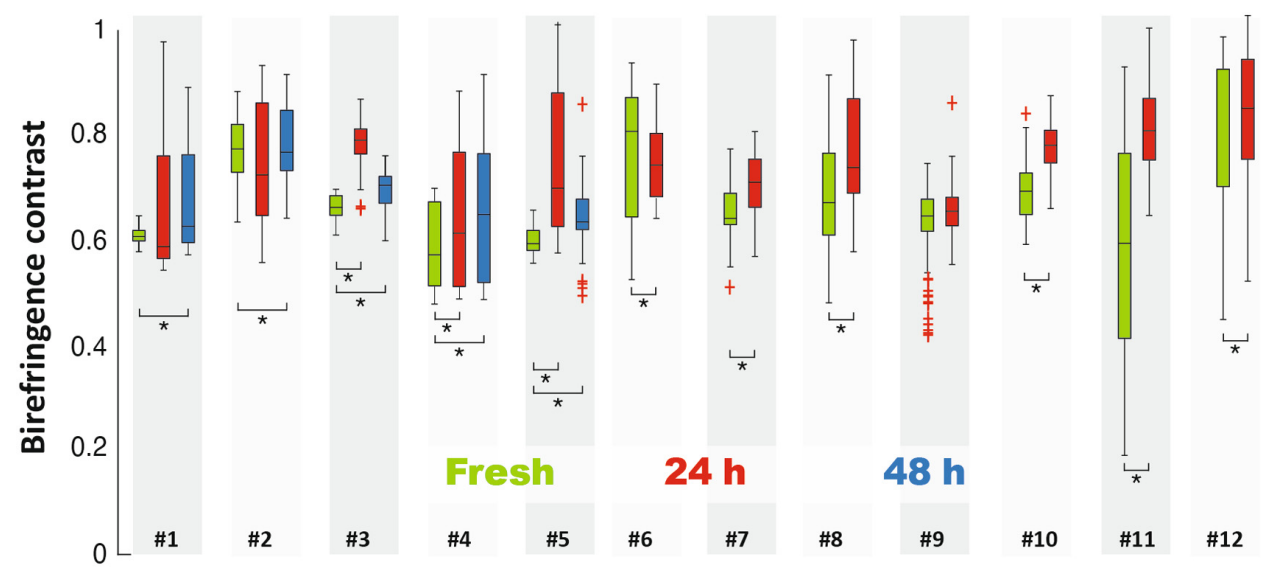

Fig. 4 Quantitative comparison of contrast change during the fixation process for all measured samples (green color represents fresh; red color, $24 \mathrm{~h}$ after fixation was initiated; blue color, $48 \mathrm{~h}$ after fixation was initiated). Red crosses represent outliers (values more than 3 times the interquartile range away from the bottom or top of the box). Asterisks indicate statistically significant differences in 11 out of the 12 samples.

\subsection{Local Birefringence Quantitative Contrast}

To provide more than the visual assessment of the effects of tissue fixation on polarization contrast, as described in Sec. 2, we applied automatic contrast analysis for both intensity and birefringence images. The results are presented as a boxplot (Fig. 4). For further statistical assessment, the paired $t$-test was applied to the results. Statistically significant differences were found for all cases except when fresh tissue was compared with $24 \mathrm{~h}$ after fixation for samples $\# 1, \# 2$, and \#9.

To provide more insight on how the contrast changes versus depth in the sample, we performed statistical (mean and standard deviation) slice-by-slice assessment of the differences in contrast between freshly measured samples and those measured 24 or $48 \mathrm{~h}$ after fixation. At each sample depth, the contrast change ( $24 \mathrm{~h}$ versus fresh and 48 versus fresh) was calculated. Depth by depth, we plot the mean contrast change over all samples with the ribbon representing standard deviation (Fig. 5). On average, we observe up to $10 \%$ increase in mean contrast to a depth of $500 \mu \mathrm{m}$ followed by up to $20 \%$ increase for deeper layers. The variation, presented as a ribbon plot, is higher at deeper layers, where the SNR is usually lower than for preceding layers.

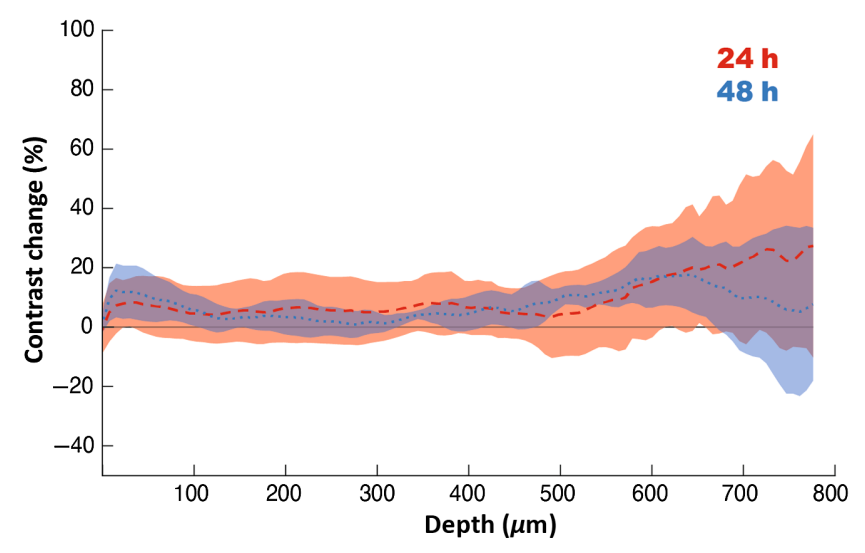

Fig. 5 Change in fixed-sample contrast relative to fresh-sample contrast versus depth. Mean contrast change versus depth is presented with red-dashed curve for changes between fresh sample and $24 \mathrm{~h}$ after fixation, and blue-dotted curve for changes between fresh sample and $48 \mathrm{~h}$ after fixation. Corresponding ribbon plots represent \pm 1 standard deviation. 


\section{Discussion and Conclusions}

In this study, the effect of fixation on local birefringence, assessed using PS-OCT, was investigated. Tissue samples from multiple locations in the oral cavity and covering a range of pathologies were imaged. Results presented in Fig. 4 show that tissue fixation does not diminish the contrast provided by the PS measurement; quite the contrary, the contrast is generally increased. Statistically significant increases in contrast were observed, compared to the contrast of fresh samples, in 11 of the 12 samples. The increase in mean contrast was calculated to be $11 \%$. The greatest increases are observed in the deepest layers, which is most likely an artifact associated with lower ANR compared to superficial layers. The observed increase in birefringence with fixation represents a positive effect enhancing the utility of fixed oral cancer tissue samples.

A limitation of our work is that it does not explain the cause of the observed changes. In this study, however, our objective was to investigate whether local birefringence contrast is preserved after tissue fixation, and the extent to which fixation permits the assessment of fixed oral cavity tissues using PS-OCT. The observed effects may be the result of tissue shrinkage, which alters the patterns of birefringent structures in the measured samples. Such contrast enhancement may arise from the fact that local birefringence is calculated from the retardance $2 k \Delta n \delta z$ across differential depth $\delta z$. In the case of axial shrinkage, differential depth $\delta z$ reduces and leads to an increase in local birefringence, other things being equal. The unexpected result of differing contrast for some samples between measurements at 24 and $48 \mathrm{~h}$ after fixation raises questions that may be answered by variable sample shrinkage and related distortion, but which requires further investigation to be confirmed or otherwise.

Promisingly, from the perspective of the utilization of fixed oral cancer tissue samples, the enhancement of birefringent contrast $C_{\Delta n}$ removes the need to image samples immediately after excision. Furthermore, such relaxation of the need to perform imaging in vivo facilitates the future use of recently developed methods, including local optic axis imaging ${ }^{25}$ and ultrahighresolution, extended-focus PS-optical coherence microscopy (PS-OCM) that cannot yet be readily moved to a dental clinic. ${ }^{30}$ PS-OCM could offer more detailed insight into fine sample structure via imaging of the local optic axis, in sample regions with sufficient local birefringence.

It must be noted that even though the results of our work justify potentially bypassing measurements of freshly excited oral cavity samples, so they could be performed later beyond the clinical setting without loss of contrast, we are not proposing to displace in vivo measurements. In fact, in vivo measurements remain our main goal and we recently reported some interesting related in vivo results from healthy volunteers. ${ }^{21}$

A corollary to the above arguments is the conclusions drawn by the study of ex vivo samples will be more challenging to apply in vivo, in which images generally show lower contrast.

In the future, the study of numerous fixed samples might lead to processing methods, further enhancement of contrast and the emergence of clear means of differentiation between normal and abnormal tissue. Our results justify the use of fixed oral tissue in seeking a deeper understanding of birefringence contrast in oral tissue pathology; however, as mentioned previously by Wood et al., ${ }^{23}$ we caution that similar investigations are advised if other tissue types are to be studied. The enhancement of contrast removes the need to image immediately postexcision and will facilitate exploration with other advanced tools, including mapping of the local optic axis with PS-OCT and ultrahigh-resolution PS-OCM.

\section{Disclosures}

The authors have no relevant financial interests in this article and no potential conflicts of interest to disclose.

\section{Acknowledgments}

The project was funded by the Australian Dental Research Foundation (Grant No. 224-2017). K. Karnowski acknowledges the support from the Polish National Agency for Academic Exchange and the European Union's Horizon 2020 research and innovation program under the 
Grant Agreement No. 666295. Q. Li acknowledges the financial support of the Australian Government International Research Training Program (RTP) Fee Offset Scholarships. M. Villiger acknowledges the support from the National Institutes of Health (No. P41EB-015903).

\section{References}

1. C. K. Lee et al., "Diagnosis of oral submucous fibrosis with optical coherence tomography," J. Biomed. Opt. 14(5), 054008 (2009).

2. N. Ozawa et al., "In vivo imaging of human labial glands using advanced optical coherence tomography," Oral Surg., Oral Med., Oral Pathol., Oral Radiol., Endod. 108(3), 425-429 (2009).

3. Z. Hamdoon et al., "Optical coherence tomography in the assessment of suspicious oral lesions: an immediate ex vivo study," Photodiagn. Photodyn. Ther. 10(1) 17-27 (2013).

4. O. R. Hughes et al., "Optical and molecular techniques to identify tumor margins within the larynx," Head Neck 32(11),1544-1553 (2010).

5. Z. Hamdoon et al., "Structural validation of oral mucosal tissue using optical coherence tomography," Head Neck Oncol. 4, 29 (2012).

6. W. Jerjes et al., "In vitro examination of suspicious oral lesions using optical coherence tomography," Br. J. Oral Maxillofac. Surg. 48(1),18-25 (2010).

7. P. Wilder-Smith et al., "In vivo diagnosis of oral dysplasia and malignancy using optical coherence tomography: preliminary studies in 50 patients," Lasers Surg. Med. 41(5), 353-357 (2009).

8. Z. Hamdoon et al., "Optical coherence tomography in the assessment of oral squamous cell carcinoma resection margins," Photodiagn. Photodyn. Ther. 13, 211-217 (2016).

9. A. M. Lee et al., "Wide-field in vivo oral OCT imaging," Biomed. Opt. Express 6(7), 2664-2674 (2015).

10. M. Kraft et al., "Optical coherence tomography: significance of a new method for assessing unclear laryngeal pathologies," HNO 58(5), 472-479 (2010).

11. P. Pande et al., "Automated classification of optical coherence tomography images for the diagnosis of oral malignancy in the hamster cheek pouch," J. Biomed. Opt. 19(8), 086022 (2014).

12. W. M. Allen et al., "Wide-field optical coherence micro-elastography for intraoperative assessment of human breast cancer margins," Biomed. Opt. Express 7(10), 4139-4153 (2016).

13. M. Villiger et al., "Deep tissue volume imaging of birefringence through fibre-optic needle probes for the delineation of breast tumour," Sci. Rep. 6, 28771 (2016).

14. F. A. South et al., "Differentiation of ex vivo human breast tissue using polarizationsensitive optical coherence tomography," Biomed. Opt. Express 5(10), 3417-3426 (2014).

15. K. H. Kim et al., "Polarization-sensitive optical frequency domain imaging based on unpolarized light,” Opt. Express 19(2), 552-561 (2011).

16. N. Gladkova et al., "Evaluation of oral mucosa collagen condition with cross-polarization optical coherence tomography," J. Biophotonics 6(4), 321-329 (2013).

17. J. Walther et al., "In vivo imaging of human oral hard and soft tissues by polarizationsensitive optical coherence tomography," J. Biomed. Opt. 22(12), 121717 (2017).

18. Y. Yoon et al., "In vivo wide-field reflectance/fluorescence imaging and polarizationsensitive optical coherence tomography of human oral cavity with a forward-viewing probe," Biomed. Opt. Express 6(2), 524-535 (2015).

19. P. Sharma et al., "Human ex-vivo oral tissue imaging using spectral domain polarization sensitive optical coherence tomography," Lasers Med. Sci. 32(1), 143-150 (2017).

20. R. Kuranov et al., "Complementary use of cross-polarization and standard OCT for differential diagnosis of pathological tissues," Opt. Express 10(15), 707-713 (2002).

21. J. Walther et al., "Depth-resolved birefringence imaging of collagen fiber organization in the human oral mucosa in vivo," Biomed. Opt. Express 10(4), 1942-1956 (2019).

22. K. Grieve et al., "Appearance of the retina with full-field optical coherence tomography," Invest. Ophthalmol. Vis. Sci. 57(9), OCT96-OCT104 (2016). 
23. M. Wood et al., "Effects of formalin fixation on tissue optical polarization properties," Phys. Med. Biol. 56, N115 (2011).

24. P. L. Hsiung et al., "Effect of tissue preservation on imaging using ultrahigh resolution optical coherence tomography," J. Biomed. Opt. 10(6), 064033 (2005).

25. Q. Li et al., "Robust reconstruction of local optic axis orientation with fiber-based polarization-sensitive optical coherence tomography," Biomed. Opt. Express 9(11), 5437-5455 (2018).

26. E. Li et al., "Three-dimensional multi-contrast imaging of in vivo human skin by Jones matrix optical coherence tomography," Biomed. Opt. Express 8(3), 1290-1305 (2017).

27. S. Prestin et al., "Measurement of epithelial thickness within the oral cavity using optical coherence tomography," Head Neck 34(12), 1777-1781 (2012).

28. S. S. Shapiro et al., "An analysis of variance test for normality (complete samples)," Biometrika, 52(3-4), 591-611 (1965).

29. M. Hollander et al., Nonparametric Statistical Methods, Wiley (1973).

30. Q. Li et al., "Vectorial birefringence imaging by optical coherence microscopy for assessing fibrillar microstructures in the cornea and limbus," Biomed. Opt. Express 11(2), 1122-1138 (2020).

Karol Karnowski is a senior researcher at Professor Maciej Wojtkowski's Physical Optics and Biophotonics Group at the PAS Institute of Physical Chemistry, working on the Imcustomeye and Create projects. For the last ten years, he has been using optical methods for early biomedical diagnostics and to investigate following disease progress by improving the contrast of the imaging techniques. He spent three years with a group at the University of Western Australia led by Professor David Sampson. He is also the leader of the project group as part of the Polish Returns program, financed by the Polish National Agency for Academic Exchange.

Martin Villiger received his MSc degree in microengineering and his $\mathrm{PhD}$ in photonics from the Swiss Federal Institute of Technology in Lausanne, Switzerland. Currently, he is an assistant professor (Harvard Medical School) at the Wellman Center for Photomedicine, located at the Massachusetts General Hospital in Boston, where he is leading research into advancing optical instrumentation for medical applications with an emphasis on polarization-sensitive and catheter-based optical coherence tomography.

Camile S. Farah is clinician-scientist and expert in oral oncological translational research. His clinical and laboratory work with Optical Fluorescence Imaging and Narrow Band Imaging has set the new gold standard for head and neck cancer endoscopy, resection margin delineation, and detection of oral potentially malignant lesions. His lab has explored diagnostic biomarker signatures for oral premalignant lesions, predictive biomarker profiles for oral cancer progression, and a multigene discriminatory biomarker panel for oral squamous cell carcinoma.

David D. Sampson is currently Pro-Vice-Chancellor, Research \& Innovation, at The University of Surrey, where he heads the research group Surrey Biophotonics. His research interests have spanned a wide range from theoretical to translational biophotonics, with strong emphasis on optical coherence tomography (OCT). He has held a long-term interest in parametric imaging, including of attenuation, elastography and polarization-sensitive OCT. He is an SPIE Fellow, OSA Fellow, IEEE Fellow, and has served on the SPIE Board of Directors as an elected member (2017 to 2019) and currently as an adviser.

Biographies of the other authors are not available. 